\title{
Security Model for a Shared Multimedia Archive
}

\author{
Karel Wouters, Brecht Wyseur, and Bart Preneel \\ Katholieke Universiteit Leuven \\ Dept. Electrical Engineering-ESAT, SCD-COSIC, \\ Kasteelpark Arenberg 10, 3001 Heverlee, Belgium \\ karel.wouters,brecht.wyseur,bart.preneel@esat.kuleuven.be
}

\begin{abstract}
Broadcasters and production houses are moving toward tape-less digital production environments for generating their multimedia content. Consequently, they are in need of a digital archive to store their new multimedia content. Ideally, this archive is closely integrated into the production environment, and enables its owners to share their content with others. In this paper, investigate the threats, associated with a shared digital multimedia archive and we describe a security model for such an archive In our approach of the model, we try to spread the security measures as wide as possible, such that single points of failure or compromise have a minimal impact on the security of the system.
\end{abstract}

\section{Introduction}

Broadcasters and production houses are moving toward tape-less digital production environments for generating their multimedia content. Consequently, they are in need of a digital archive to store their new multimedia content. Examples of efforts in this direction include the BBC, ZDF and RAI digital archives. Ideally, such an archive is closely integrated into the production environment. This not only facilitates the archiving itself but it also enables faster access to and easier re-use of archived content. Moreover, it enables efficient disclosure to the general public, because the content is readily available to be converted from a highresolution version to a customer-suitable format, as can be seen from the BBC Motion Gallery initiative [1].

In the IPEA [2] project, one of the goals was the design of an architecture for a multimedia archive, equipped with an extensive meta-data model, such that the archive can be shared easily using a subset of the meta-data model, among several Broadcasting Companies (BCs) and production houses. ${ }^{1}$ An archive can be deployed in the exist-

\footnotetext{
${ }^{1}$ The BCs in the IPEA project produce a part of their material by them-
}

ing production environment of $\mathrm{BCs}$, but also in that of an Archive Service Provider (ASP). In the latter case, the ASP provides storage facilities and software to enter, retrieve and document multimedia content from the archive, as well as the facilities (production environment) to produce new content, using the archive. This allows small BCs to completely outsource the maintenance of the archive and the production environment, while big BCs can use these services to provide an environment for production houses, attached to them. A Media Asset Manager (MAM), such as included in [3], is at the centre of our model. The MAM is a set of programs that acts as a portal to the archived content. It is used by the archivists to add meta-data to multimedia files and by other users to search, retrieve and add multimedia content. Every regular action on archived content should be performed through the MAM.

While this vision of a shared archive generates interesting business opportunities in the production environment, it also generates challenging security threats. The security model that we propose in this paper is designed to resist these specific threats, and to resolve the main concern for the industrial partners in the project: controlling the access to and usage of the multimedia material of a $\mathrm{BC}$ within the archive, even if it is located within an ASP.

While our design has some resemblance with existing DRM architectures and implementations, such as the Windows Media Rights Manager or the architecture proposed in the OMA DRM 2.0, we were confronted with a very specific environment. In the classical DRM setting, the intended audience is not known in advantage, has a rather large set of privacy rights, and content is uploaded to platforms out of the control of the content owner. The focus of such a system is on distributing content to customers, rather than archiving high-value, high-resolution original work. Although some aspects of digital archiving for multimedia content have been thoroughly investigated, security

selves, sometimes subcontracting a production house. In a more general setting, the this task can be outsourced completely to production houses. 
issues seem to have been neglected a little bit. One reason may be that for long-term archiving, confidentiality of secured content cannot be guaranteed. Another reason is because content producers have been moving to digitallyaccessible archiving only recently, and sharing happened by tape-handovers. Because of the limited number of software and hardware providers in this niche market, new functionality is only developed when content providers explicitly ask for it.

The remainder of this paper is structured as follows: in section 2, we discuss some the specific threats mentioned above. The functional components in the model are presented in section 3, followed by an illustration of the actual information flow, in three usage scenarios in section 4 . We conclude with some reflections on the overall security of the proposed model.

\section{Assets and Threats}

In the archive type, described above, we can define assets to be protected and threats that jeopardise those assets. Threats are translated into risks if they can be exploited by a potential attacker. Below, we list the important assets in the archive and the threats to which they are exposed.

\section{$2.1 \quad$ Assets}

From our discussions with the industrial partners in the IPEA project, we learnt that the following assets are of high importance:

The production environment This is an essential asset; it should be available at all times. The production environment consists of the hardware on which the $\mathrm{BC}$ handles multimedia content, the network that connects it, and all servers that offer services to maintain it.

Integrity of archived material including meta-data If meta-data or multimedia content is corrupted (changed), it becomes useless [4], especially in the case of news items. The integrity property and the ability to prove integrity are assets as they make a collection of material more valuable.

Knowledge of meta-data and meta-data structures Adding meta-data to multimedia content is a timeconsuming activity, so meta-data itself is valuable.

Scheduling data Long-term scheduling data is strategic information, both for commercial and public broadcasters.
Intellectual property Content produced by or exclusively for a BC should be protected against unauthorised re-use by others. Moreover, content on which time- and location-restricted licenses were bought by the BC, should be protected properly to avoid legal cases against the BC.

The threats, listed below, should be considered with these assets in mind. We assumed that the attacker's goals are as broad a possible, ranging from just being as destructive as possible to stealing IP or content on which the BC has limited rights.

\section{$2.2 \quad$ Threats}

Below we summarise the most relevant threats for a shared digital archive. Note that threats can originate from inside or outside the ASP or BC networking environment. Furthermore, in the ASP environment, the system administrators are not necessarily fully trusted by the BCs. The consequence is that threats in the ASP environment bear greater risk.

\subsubsection{Generic threats}

Generic threats exist independently from the model. They include threats on the physical infrastructure and on the users themselves, e.g. power outages, natural disasters, extortion, social engineering, bribing. These threats should be protected against by company policies, insurances and proper user education.

Apart from these threats, there also exist some threats to generic components (servers and infrastructure) on which the model relies. E.g., a Denial-of-Service (DoS) attack can always be mounted if enough components are blocked or overloaded. This is not a consequence of the specific construction of the archive. Therefore, we assume that the network and generic servers are protected by default.

\subsubsection{Threats to the model}

The following threats are specific to the model and need extra attention if an implementation of it is deployed.

Breaking into components For this threat, we assume that the attacker gains full access to the targeted component.

Spoofing components When an attacker spoofs a component, he mimics the behaviour of the component in the archive, by replacing the component with his own.

Breaking into communications We distinguish two types of this threat: unauthorised read access to information 
being transmitted and secondly, careful changes or interception of traffic between components; such that unwanted behaviour occurs.

Usage threats Finally, malicious users can try to use the system in such a way that the resources are exhausted, e.g. adding random junk to the archive.

\subsubsection{Consequences}

The possible consequences of the threats mentioned vary in severity. When the archive is tightly embedded into the production environment, a denial of service can cause a BC to stop broadcasting. Furthermore, tampered meta-data can make the archive unsearchable and hence useless. Unauthorised access to high-res content is also quite serious, and using an ASP, sharing the same physical archive with other $\mathrm{BCs}$, only elevates the risk. For high-res content on which the $\mathrm{BC}$ only has limited rights (e.g. it can only broadcast a certain series in a given time window), the owner might take legal action if he would spot his content on the Internet, or on pirated DVDs.

Secondary consequences are that (strategic) broadcasting schemes and sets of meta-data could be leaked, such a $\mathrm{BC}$ might learn which kind of content his competitors are producing and how their content is documented.

\section{Model}

In this section, we describe the structure and components of our security model.

In our model, depicted in Figure 1, we try to spread the security measures as wide as possible, such that single points of failure or compromise have a minimal impact on the security of the system. The components in our security model can be deployed in the ASP as well as in the BC (see Figure 1):

Applications servers - MAM The MAM is the entry point for the user to the multimedia archive. It communicates with the Authorisation Server to check the user's permission on meta-data of a given multimedia file. It also connects to the Storage Manager to initiate transfers of high-res content. It is the only regular access channel to the Metadata Database.

Entity authentication servers - Active Directory (AD) The AD in the $\mathrm{BC}$ should contain all roles, relevant to the MAM system. The MAM completely relies on the AD for its user authentication. Moreover, the BC sets up an authentication webservice, such that users can retrieve credentials from the $\mathrm{AD}$ of their employing organisation, when using an ASP's infrastructure.
For the AD in the ASP, we wanted to avoid double user management. Therefore, the AD in the ASP should only contain guest accounts with minimal privileges, which can be adjusted after authentication of the user by his employer (the BC).

Storage Manager The Storage Manager is the only interface to the multimedia archive storage facilities for high-res content (the Archive); it stores new content and retrieves existing content, if the user in front of the MAM is allowed to do so. The Storage Manager interacts with the Editing/Ingesting Workstations to retrieve and store content.

Because all high-res content in our model remains encrypted outside Editing/Ingesting Workstations, there must be a means to transport decryption keys to these workstations. This is done using licenses, which can be packed together with the encrypted high-res material. The Storage Manager retrieves this from a License Server, given the Unique Material Identifier (UMID) and the users ID.

License Servers These servers issue licenses that describe permissions of a user on a piece of multimedia content. The license for a given UMID is derived from the rules in the Rules Database, and also contains the key to decrypt encrypted content (the Content Encryption Key, $K_{C}$ ). The set of permissions is signed, together with the user ID and the UMID, using the private key $\operatorname{Pr}_{L}$ of the License Server. The Content Encryption Key $K_{C}$ is encrypted under the public key $\mathrm{Pu}_{W}$ of the Workstation on which the content is going to be uploaded. Optionally, the license itself can be encrypted, using the public key $\mathrm{Pu}_{U}$ of the user for which the content is intended. This ensures that even compromised Workstations cannot get access to high-res content without the user ever being logged in.

Authorisation Servers These servers determine whether or not a user has access to certain meta-data, browsable content or high-res versions of the content. The rules, specifying the access conditions are stored in the Rules Database.

Editing/Ingesting Workstations On Editing Workstations, existing content is stored by the Storage Manager to be edited by the user. The Ingesting Workstations are used to upload new content to the archive, through the Storage Manager. This is the only place in our model on which decrypted high-resolution content is available. In all other circumstances, high-res material is only available in encrypted form. Editing/Ingesting Workstations have an encryption key pair $\left(\mathrm{Pu}_{W}, \operatorname{Pr}_{W}\right)$, such that content encryption keys can be sent to them without being compromised. In previous work [6], we have described specialised mitigation techniques that can be deployed to protect these keys and the unencrypted content. 


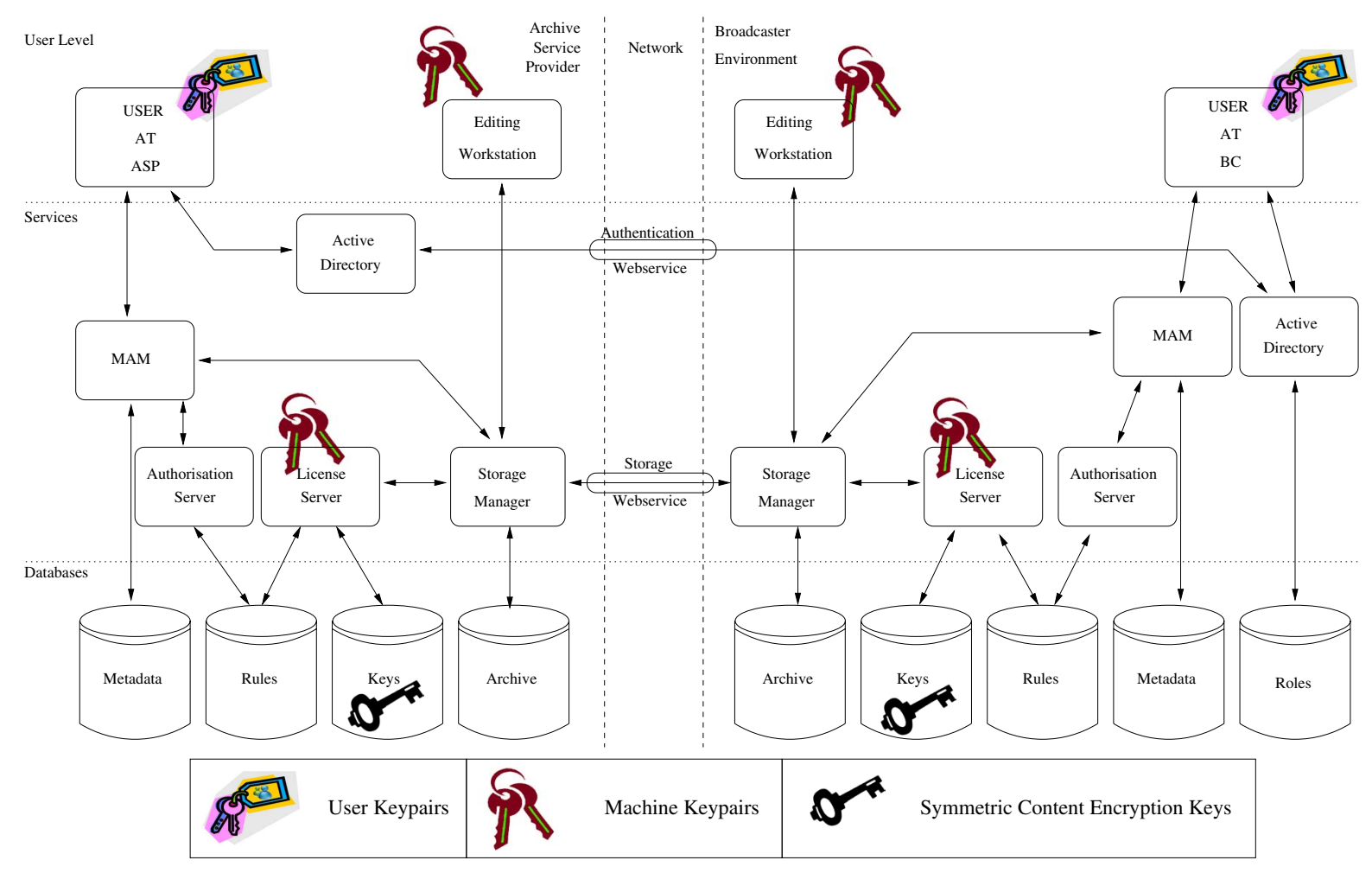

Figure 1. Security Model Overview

Key Databases Key Databases hold the content encryption keys $K_{C}$ for all multimedia content that is stored in the entity in which the key server is deployed. These keys are symmetric keys and should be securely backed up. Access to these databases should be tightly controlled; License Servers that access these databases should access them over a secure, authenticated channel that is set up after proper authentication of the License Sever, using his key pair $\left(\mathrm{Pu}_{L}, \mathrm{Pr}_{L}\right)$.

Meta-data Databases In the Meta-data Database, an ASP will hold only information that applies to the content he serves, while a BC holds at least all meta-data about content on which she has the intellectual property (IP). This can be complemented with meta-data of content on which the $\mathrm{BC}$ has or might obtain usage rights (e.g, content from other BCs). This implies that BCs can hold meta-data on content they do not own or have read access to.

Rules Databases These databases hold the rules for the License Server and the Authorisation Server. An ASP stores only the rules regarding the content he serves, while a $\mathrm{BC}$ stores all rules that apply to content on which she has the IP, complemented with the rules of content on which the $\mathrm{BC}$ has usage rights. The rule-set in an ASP, covering the multimedia content of a certain $\mathrm{BC}$, is always a subset of the rule-set in the Rules Database of that BC. Read access to the rules database happens through the Authorisation Server and the License Server. Write access happens through the MAM, automatically when a new item is added, or by owners and rules administrators that want to restrict or extend rules.

High-resolution multimedia file storage - the Archive These storage facilities for high-res multimedia material are sometimes referred to as the "Archive". For a small BC, the storage for the multimedia content might consist of hard disks, while a large $\mathrm{BC}$ will resort to a tape library. Note that the Archive contains only encrypted versions of highresolution content.

Remote functionality BCs may need access from within their facilities to content they own, stored by an ASP. Therefore, we foresee servers that run web services, which are deployed at all parties, participating in the archive. This way, remote authentication, remote access and sharing becomes possible. In essence, they expose the functionality of the model to an external network. These services can be tightly controlled by content-inspecting firewalls, and may only be accessible through dedicated connections. 
Machine authentication To ensure that functionality is only exposed to those services that are entitled to use it, all servers in the network are equipped with a keypair to identify themselves. Authentication and encryption can then happen over SSL or even at application layer, using Web Services Security [5].

User The users in our model are people, working for a $\mathrm{BC}$. They can be employed directly by the BC, or through a subcontractor. They can be located in a BC's network or in an ASP's network; role-based access control regulates what users can do.

Keys In our model, three kinds of keys are used. Content encryption keys $K_{C}$ are symmetric keys that are used to encrypt high-res multimedia content. They are generated by the License Server and stored in a Key Database. Keypairs for users are issued such that user-specific licenses can be generated. Keypairs for machines to enable a trust relations ship between different components, and to generate signed licenses with embedded encrypted content keys.

\section{Usage Scenarios}

In this section we clarify the components listed above by specifying how they behave in some typical usage scenarios.

\subsection{Access to multimedia content from within the ASP}

This usage scenario describes how employees or subcontractors of BCs can use the multimedia content which their employer owns or has read access to, when they are working within a production environment, offered by an ASP. The Archive that is part of this environment hosts encrypted multimedia content of several BCs, while the user authentication is provided by the home organisation (the employing $\mathrm{BC}$ ): the user logs on to the ASP system as a guest (Figure 2, step 1a), and is presented with a browser to select the $\mathrm{BC}$ that employs him, after which he is redirected to a web page of that $\mathrm{BC}(1 \mathrm{~b})$. After user authentication, the $\mathrm{BC}$ issues a credential that is imported by the ASP's AD and that elevates his local privileges on BC's content (1c), by adding the user to a previously defined $\mathrm{BC}$ group. This group membership should be discarded when the user logs out.

The user accesses the archive using the MAM (2), and queries the Meta-data Database through it (3). The results are retrieved from the Meta-data Database and passed to the Authorisation Server (4a) who determines which results are accessible by this user (4b). To facilitate this, metadata can optionally be subdivided in security classes. The

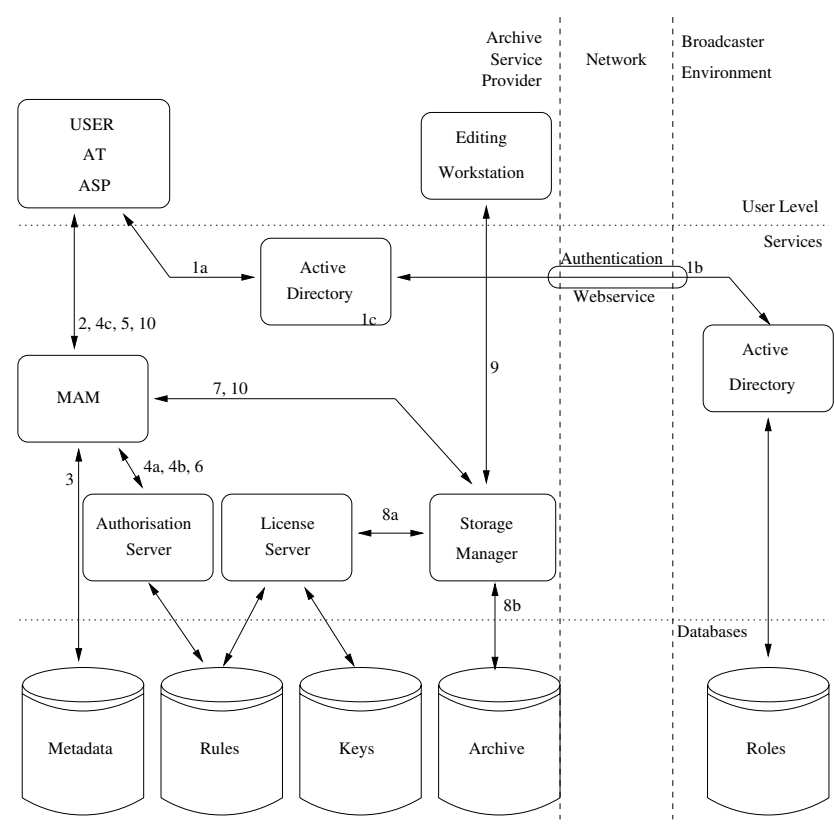

Figure 2. Retrieving content from within the ASP's production environment

MAM presents the filtered set of meta-data, together with the rights (view, re-use, etc.) the user has on the multimedia content they document (4c).

After having seen the result set, the user selects one or more items that he wants to use, and asks the MAM to put them on an Editing Workstation (5). The MAM then checks if the user has the proper privileges to actually use the content (6), and generates a request for the Storage Manager to put the content on a workstation for the given user (7).

Upon receiving this request the Storage Manager contacts the License Server to provide a license for the requested content (8a). This license contains a signed list of permissions that the user has on the given content, and the key $K_{C}$ to decrypt the content, encrypted under the public key $\mathrm{Pu}_{W}$ of the Workstation on which the content will be uploaded. Optionally, the signed license is encrypted with the public key $\mathrm{Pu}_{U}$ of the user. If a license is returned and contains at least read privileges, the Storage Manager retrieves the encrypted multimedia file from the Archive (8b), stores it on an Editing Workstation together with the license (9), and provides the user with the location of the file (10).

The user can now access the Editing Workstation, and use his private key $\operatorname{Pr}_{U}$ to decrypt the high-res content. The Workstation then checks the signature on the license, using the public key $\mathrm{Pu}_{L}$ of the License Server, and decrypts the content Encryption Key $K_{C}$ with his private key $\operatorname{Pr}_{W}$. The software, running on the Workstation, will only allow the user to perform only those actions, listed in the license. 


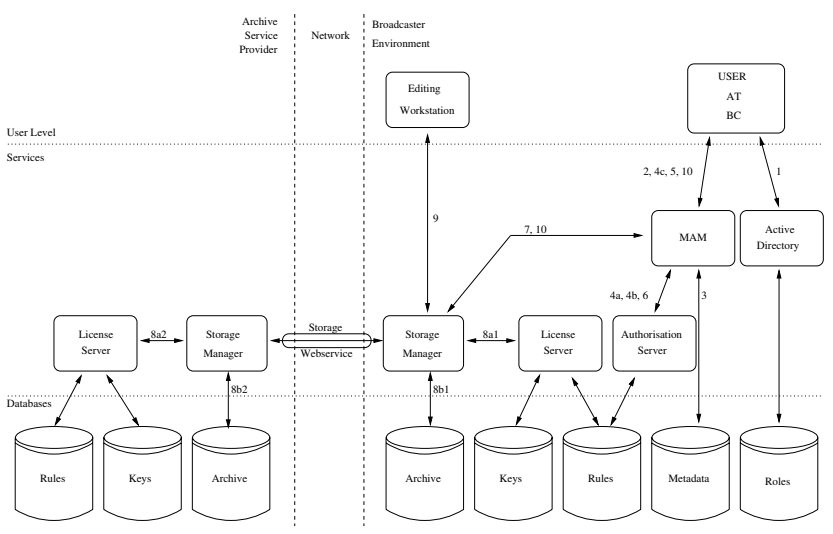

Figure 3. Retrieving content from within the $B C$ 's production environment

\subsection{Access to multimedia content from within the $\mathrm{BC}$}

The next usage scenario describes how employees of $\mathrm{BCs}$ can use the multimedia content which their employer owns, when they are working within their own production environment. The Archive that is part of this environment hosts encrypted multimedia content owned by the BC itself, which is considered to be to sensitive to store in the ASP. Another reason might be that the content must be located as close as possible to the $\mathrm{BC}$ for production reasons.

Other multimedia content can reside in the ASP. This can be less sensitive content, content that the $\mathrm{BC}$ wants to share with others or content shared by another BC.

User authentication happens in the regular way: authentication to the BC's system using his own credentials. The other steps are similar, except for the actions of the Storage Manager. When the Storage Manager receives a request from the MAM to put content for a certain user on an Editing Workstation (7), he first checks where the content is stored. If the content is stored in the BC itself, similar steps as in the previous scenario are executed (8a1, 8b1). If the content resides in the ASP, the Storage Manager of the $\mathrm{BC}$ forwards the request to the Storage manager of the ASP (8a2), which returns the content together with a license. Note that information about the user's identity or group membership has to be passed to the License Server of the ASP, together with the public key of an Editing Workstation, for the License Server to be able to generate a license that is usable in the BC's context. Steps (9) and (10) are similar to those of the previous scenario.

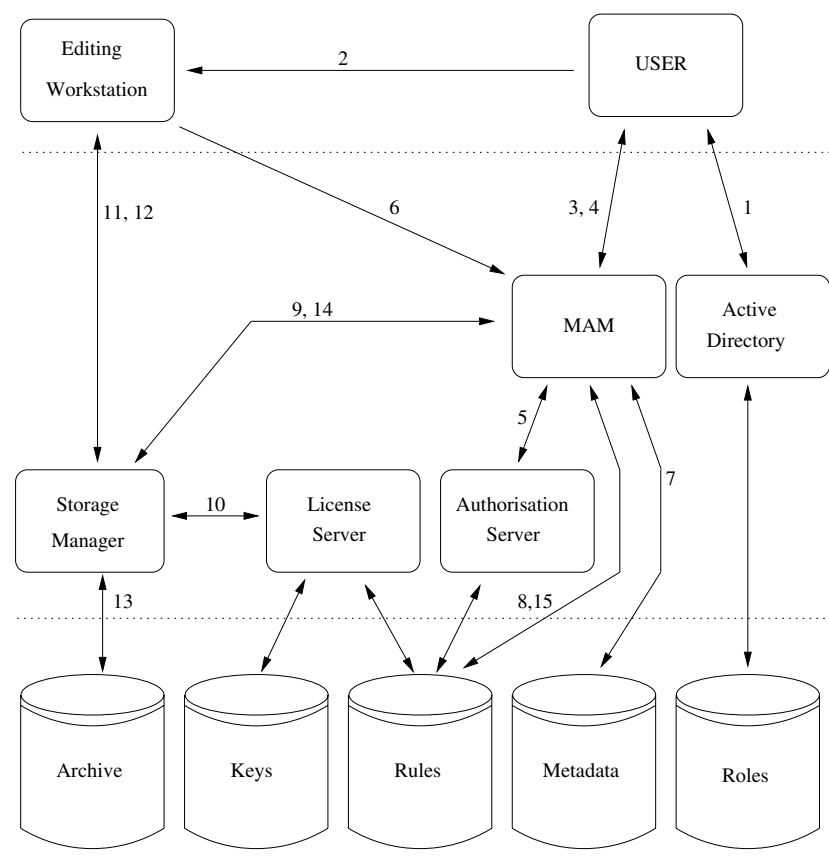

Figure 4. Ingesting content from within the $B C$ 's production environment

\subsection{Adding multimedia content to the archive}

In this usage scenario, we describe how new content is to be added to the archive. This scenario is the same in the ASP and the BC, with the slight difference that in the ASP, new information from the Rules and Meta-data Databases has to be added to the corresponding databases in the $\mathrm{BC}$ that owns the content. Another difference is the login procedure, which is illustrated in the two usage cases above. We present the scenario from within the BC.

When a user generates new content, possibly using fragments of archived material, he stores it on an Ingest Workstation $^{2}$ (step 2 in Figure 4), after having authenticated (1). Then, he starts the MAM (3), and indicates that he has a new item to add to the archive by referring to the place $L$ on the Ingest Workstation (4) on which the content resides. After checking if the user is allowed to add new content (5), the MAM retrieves the Unique Material Identifier (UMID) of the content to be added, together with some meta-data that can be generated automatically, e.g. lenght, keyframes, a browsable copy (6). The user's credentials are used to retrieve these data.

Then, the MAM generates a new item in the Meta-data Database (7) and a set of rules in the Rules Database (8).

\footnotetext{
${ }^{2}$ The Ingest Workstation may also serve as an Editing Workstation, on which the content was generated
} 
One of the rules indicates that the content itself is not yet present in the Archive and it allows the user to add the actual file. Other rules could be generic ones, giving access to meta-data and the content itself for each group member of the user's BC primary group.

The MAM presents the user's credentials, UMID, and $L$ to the Storage Manager, asking him to add the content to the Archive (9). Then, the Storage Manager uses the credentials, the location $L$ and the UMID to retrieve a license from the License Server (10). This license indicates that the content is to be added to the Archive, together with the Content Encryption Key $K_{C}$, secured by the public key $\mathrm{Pu}_{W}$ of the Ingest Workstation.

The license is forwarded to the Ingest Workstation (11), which extracts the key $K_{C}$ using its private key $\operatorname{Pr}_{W}$, encrypts the multimedia content, and passes it on to the Storage Manager (12). The Storage Manager enters the encrypted content into the Archive (13), and notifies the MAM that it has done so (14). The MAM removes the special rule from the Rules Database (15), and may send a notification to archivists and rules managers that new content is available.

When operating from within an ASP, one additional step is needed: upon creation of the new item, meta-data and rules regarding the item have to be copied to the databases of the BC.

\section{Conclusion}

In this paper, we presented a security model, that can be used to design an architecture for a shared multimedia archive. We made the following working assumption: a BC wants to store some of its content in a series of ASPs. He wants to share a subset of that content with others; the rest is for users working for the $\mathrm{BC}$, at the ASP's location. Moreover, the main security concern of the $\mathrm{BC}$ is illegitimate access to high-resolution content and the availability of the production environment.

The model is quite safe because of the distribution of power: In almost every case, at least two separate components of the model have to be compromised in order for an attacker to gain illegitimate access to high-resolution content. There are two notable exceptions to this: illegitimate write access to the Rules Database can raise the privileges of existing users on any object. Secondly, ultimate control of an Ingest/Editing workstation gives the attacker access to every object, stored on that workstation.

In future work, a more fine-grained threat analysis could be conducted, based on an actual architecture and possibly an implementation of it. Note that the software products for implementing an archive in the broadcasting world do not allow for a non-trivial adaptation such that they follow our model.
Whatever the output of this analysis might be, the production process for broadcasting companies will move from tapes to online digital archives and the benefits of efficiency and flexibility must be complemented by a sufficient level of control over access to and usage of the stored multimedia content. This work is a first step toward an integrated approach to do so.

\section{Acknowledgements}

The authors are grateful to Koen De Hauw for his help during our work, and providing us valuable insights in the daily operation of a content producer.

This work has been partially funded by the IBBT/IPEA project, a Ph.D. grant of the Institute for the Promotion of Innovation through Science and Technology in Flanders (IWT-Vlaanderen), and by the BCRYPT Interuniversity Attraction Pole (IAP VI/26) programme of the Belgian government.

\section{References}

[1] BBC Motion Gallery. http://www. bbcmotiongallery.com.

[2] The IBBT/IPEA Project - Innovative Platform for Electronic Archiving. http://projects.ibbt.be/ ipea.

[3] Ardendo AB. Ardome Archive Edition - System Architecture, version 1.0a edition, June 2004.

[4] Kenny Irby. L.A. Times Photographer Fired Over Altered Image. Poynter Online, http://www . poynter.org, QuickLink: A28082, April 2003.

[5] Kelvin Lawrence and Chris Kaler. OASIS Web Services Security (WSS) TC. http: //www.oasis-open.org/committees/ tc_home.php?wg_abbrev=wss.

[6] Brecht Wyseur, Karel Wouters, Mina Deng, Thomas Herlea, and Bart Preneel. On the design of a secure multimedia archive. In 1st Benelux Workshop on Information and System Security (WISSec 2006), page 14, Antwerp,BE, 2006. 